\title{
Processing of 3D weather radar data with application for assimilation in the NWP model
}

\begin{abstract}
The paper is focused on the processing of $3 \mathrm{D}$ weather radar data to minimize the impact of a number of errors from different sources, both meteorological and non-meteorological. The data is also quantitatively characterized in terms of its quality. A set of dedicated algorithms based on analysis of the reflectivity field pattern is described. All the developed algorithms were tested on data from the Polish radar network POLRAD. Quality control plays a key role in avoiding the introduction of incorrect information into applications using radar data. One of the quality control methods is radar data assimilation in numerical weather prediction models to estimate initial conditions of the atmosphere. The study shows an experiment with quality controlled radar data assimilation in the COAMPS model using the ensemble Kalman filter technique. The analysis proved the potential of radar data for such applications; however, further investigations will be indispensable.
\end{abstract}

Keywords

Weather radar $\cdot$ radar reflectivity $\bullet$ data quality $\bullet$ data assimilation

(C) University of Warsaw - Faculty of Geography and Regional Studies
Katarzyna Ośródka ${ }^{1}$, Jan Szturc ${ }^{2}$, Bogumił Jakubiak ${ }^{3}$, Anna Jurczyk ${ }^{4}$

'Department of Ground Based Remote Sensing Institute of Meteorology and Water Management National Research Institute

e-mail: Katarzyna.Osrodka@imgw.pl

${ }^{2}$ Department of Ground Based Remote Sensing Institute of Meteorology and Water Management National Research Institute

e-mail: Jan.Szturc@imgw.p

${ }^{3}$ Interdisciplinary Centre for Mathematical and

Computational Modelling

University of Warsaw

e-mail: B.Jakubiak@icm.edu.pl

${ }^{4}$ Department of Ground Based Remote Sensing

Institute of Meteorology and Water Management

National Research Institute

e-mail: Anna.Jurczyk@imgw.pl

Received: 10 February 2014

Accepted: 30 April 2014

\section{Introduction}

The paper presents the algorithms for both the correction and quality characterization of $3 \mathrm{D}$ reflectivity data. The processed radar data was applied to the experiment with data assimilation in the NWP model using a filtering method.

\section{Radar data quality issues}

The weather radar has become a more and more common tool to provide input data for many applications, such as rainfallrunoff modelling, assimilation in NWP models and air pollution dispersion. Observations are available with very a high time and space resolution over a large area which makes them highly valuable. However, the data is burdened with a number of errors from different sources, which have been comprehensively discussed by many authors (e.g. Meischner 2004; Šálek et al. 2004; Michelson et al. 2005; Villarini \& Krajewski 2010). Hardware sources of errors are related to the instability of electronics, antenna accuracy and signal processing accuracy (Gekat et al. 2004). Other nonmeteorological errors result from electromagnetic interference with sun and external microwave emitters, attenuation in rain, ground clutter (Germann \& Joss 2004), anomalous propagation of the radar beam due to specific atmosphere temperature or moisture gradient (Bebbington et al. 2007), shielding due to topography (Bech et al. 2007) or by nearby objects such as trees and buildings, and echoes from birds and insects. The next group of errors is associated with scan strategy, radar beam geometry and the broadening of the beam width with distance from the radar site.

Thus quality control to ensure reliable data is a challenging task. At the same time information on measurement uncertainty is becoming essential, especially for end-users, so much research effort is currently being focused on quality issues (e.g. Einfalt et al. 2010; Zappa et al. 2010).

\section{Radar data assimilation in NWP models}

Data assimilation as a major component of numerical weather prediction (NWP) models consists of the process of creating the best estimate of the initial atmospheric state. It involves combining all sources of information from the forecast model itself as the first guess, together with multi-source observations and the associated uncertainties.

The current progress in computational possibilities is enabling the development of models with higher spatial resolution, which require the provision of data describing smaller scale phenomena, e.g. convective processes. Radar reflectivity data of high temporal (about 5-10 $\mathrm{min}$ ) and spatial resolutions (mostly $1 \times 1 \mathrm{~km}$ ) assimilated into the NWP model can improve the initial description of the atmospheric state (Sun 2005). A number of papers show that the assimilation of radar-derived data (reflectivity or Doppler data) in NWP models has the potential to improve precipitation forecasts, especially to better represent convective processes in the atmosphere.

Weather radar data can be assimilated in NWP models using different approaches, such as nudging, variational analysis, and ensemble Kalman filtering. The ensemble Kalman filter (EnKF) method transforms the analysis ensemble of model states from one to the next analysis time using the sample mean and covariance of the resulted ensemble as the background mean and covariance for the next analysis (Houtekamer et al. 2005; Xue et al. 2010). 
Radar data processing

The weather radar data employed in the investigations was generated by the Polish radar network POLRAD operated by the Institute of Meteorology and Water Management - National Research Institute (IMGW-PIB). At present, POLRAD consists of eight C-Band Doppler radars of Selex SI Gematronik, two of which are polarimetric. The standard range of the measurements is $250 \mathrm{~km}$.

The weather radar data should be corrected at the earliest stage of data processing, i.e. on the 3D data volumes before it is transformed into various $2 \mathrm{D}$ products. Our work is focused on the volumes of radar reflectivity $Z$ (in dBZ) (Ośródka et al. 2014). The corrections are determined by considering particular quality factors (such as the existence of non-meteorological echoes, beam blockage, and attenuation in rain), described in this section in more detail. Simultaneously, data quality in terms of a particular factor is characterized by means of quality index $(Q I)$ - a unitless quantity which ranges from 0 for totally unusable data to 1 for excellent data (Einfalt et al. 2010). The most employed algorithms are empirically established and require the optimization of a number of parameters and thresholds. Next, total QI is computed from all individual Q/s using the multiplicative scheme. The quality field is assigned to the radar data as useful additional information for different applications, such as assimilation to the NWP model.

\section{Horizontal and vertical broadening of a radar beam}

Radar measurements assigned to successive measurement points (so called gates) along radar beams are determined for certain surrounding areas defined by beam width and pulse length. However, the actual physical beam width increases with range, so that the measurement comes from a larger pulse volume, which results in the inevitable increase of averaging errors.

The horizontal and vertical broadenings $L_{H}$ and $L_{V}$ of a vertical cross-section through the radar beam can be calculated for each gate by the formulae (Fig. 1):

$L_{H}=\left(l+\frac{P_{L}}{2}\right) \cdot \cos \left(\varepsilon-\frac{\varphi}{2}\right)-\left(l-\frac{P_{L}}{2}\right) \cdot \cos \left(\varepsilon+\frac{\varphi}{2}\right)$

$L_{V}=\left(l+\frac{P_{L}}{2}\right) \cdot \sin \left(\varepsilon+\frac{\varphi}{2}\right)-\left(l-\frac{P_{L}}{2}\right) \cdot \sin \left(\varepsilon-\frac{\varphi}{2}\right)$

where $\varepsilon$ is the elevation angle, $/$ is the distance to the radar site (in $\mathrm{km}), \varphi$ is the beam width, and $P_{L}$ is the measurement gate length related to radar pulse length (in $\mathrm{km}$ ).

For both quality indices it is assumed that the quality is perfect for the relevant quality indicator below the preset threshold, then decreases linearly to achieve zero for the indicator above the second threshold. Finally, $Q I_{B R O A D}$ is calculated as the product of these two indices:

$$
Q I_{B R O A D}=Q I_{L H} \cdot Q I_{L V}
$$

Removal of geometrically-shaped non-meteorological echoes (spike)

The spurious echoes from the sun and interfering signals from external antennas (e.g. RLAN), so-called spikes, are nonmeteorological echoes often contaminating radar data. They are characterized by a specific spatial structure that clearly differs from the precipitation field pattern: they resemble spikes along the whole or a large part of a single or a few neighbouring radar beams. The removal procedure is not very difficult unless the spike interferes with a precipitation field. The algorithm developed for the removal of such artefacts identifies spikes, cuts them out from the precipitation field, and replaces them with the proper reflectivity values.

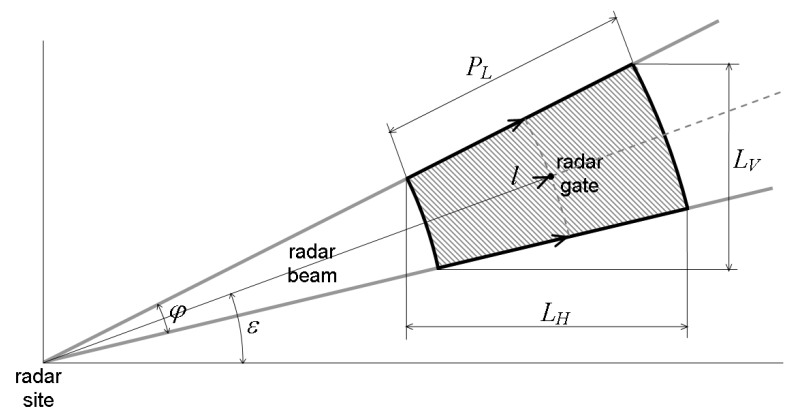

Figure 1. Vertical cross-section through radar beam

Two sub-algorithms dedicated to spike removal are introduced: for "wide" and "narrow" types of spikes (Fig. 2).

In the algorithm for "wide" spike detection the spatial structure of a radar echo is analysed. For a given gate, locally determined reflectivity variances across and along the radar beam are examined, and a given echo is classified as a potential spike if the first variance is high whereas the latter is low:

$$
\begin{aligned}
& {\left[\operatorname{var}_{\text {across }}(Z(\alpha, l)) \text { is high }\right] \text { and }\left[\operatorname{var}_{\text {along }}(Z(\alpha, l)) \text { is low }\right] \Rightarrow} \\
& \Rightarrow \text { potential "wide" spike in }(\alpha, l)
\end{aligned}
$$

where $\alpha$ is the azimuth, $I$ is the distance to the radar site, var ${ }_{\text {across }}$ is the variance calculated across the radar beam within a few azimuths; var $_{\text {along }}$ is the variance along the radar beam at a distance up to \pm several $\mathrm{km}$.

For each radar beam, if the fraction of gates classified as potential spikes is higher than $45 \%$, then these gates change their status into "spike" and the whole beam is treated as corrupted by a spike. The quality index $Q I_{\text {SPIKE }}$ for all its gates is decreased to 0.5 .

The next algorithm is focused on "narrow" spikes (with azimuthal width up to $\left.7^{\circ}\right)$. For each gate $(\alpha, l)$ with echo detected, gates at neighbouring beams of azimuths $\alpha \pm d$ at the same distance to the radar site / are tested:

$[Z(\alpha, l)-Z(\alpha+d, l)>10 \mathrm{dBZ}]$ and $[Z(\alpha, l)-Z(\alpha-d, l)>$

$>10 \mathrm{dBZ}] \Rightarrow$ potential "narrow" spike in $(\alpha, l)$

The procedure is repeated for $d-1, \ldots, 1^{\circ}$. Finally, if the fraction of potential gates in the beam exceeds $25 \%$ then these gates change their status into "spike" and for the whole beam the quality $Q I_{\text {SPIKE }}$ decreases to 0.5 .

When detection of both types of spikes is completed the contaminated gates are interpolated with the average reflectivity of meteorological echoes from neighbouring gates (left- and rightmost) at the same distance to the radar site.

\section{Removal of non-meteorological echoes}

The algorithm is designed to detect and remove nonmeteorological echoes existing at low and very high altitudes (Fig. 3). The first sub-algorithm is to find artefacts with weak echoes at low altitudes caused by different interferences like side-lobes that hit the ground close to the radar site, anomalous propagation, and biological objects (flocks of birds, swarms of insects). The analysis is based on two relevant detectors of nonmeteorological echo, $u_{z}$ and $u_{h}$, which have been empirically optimized on historical data. The detectors are defined as unitless quantities determined from linear functions of radar reflectivity and altitude of the echo in the given gate, respectively. If for the gate the product of the detectors exceeds the preset threshold, then the echo is considered false and removed: 


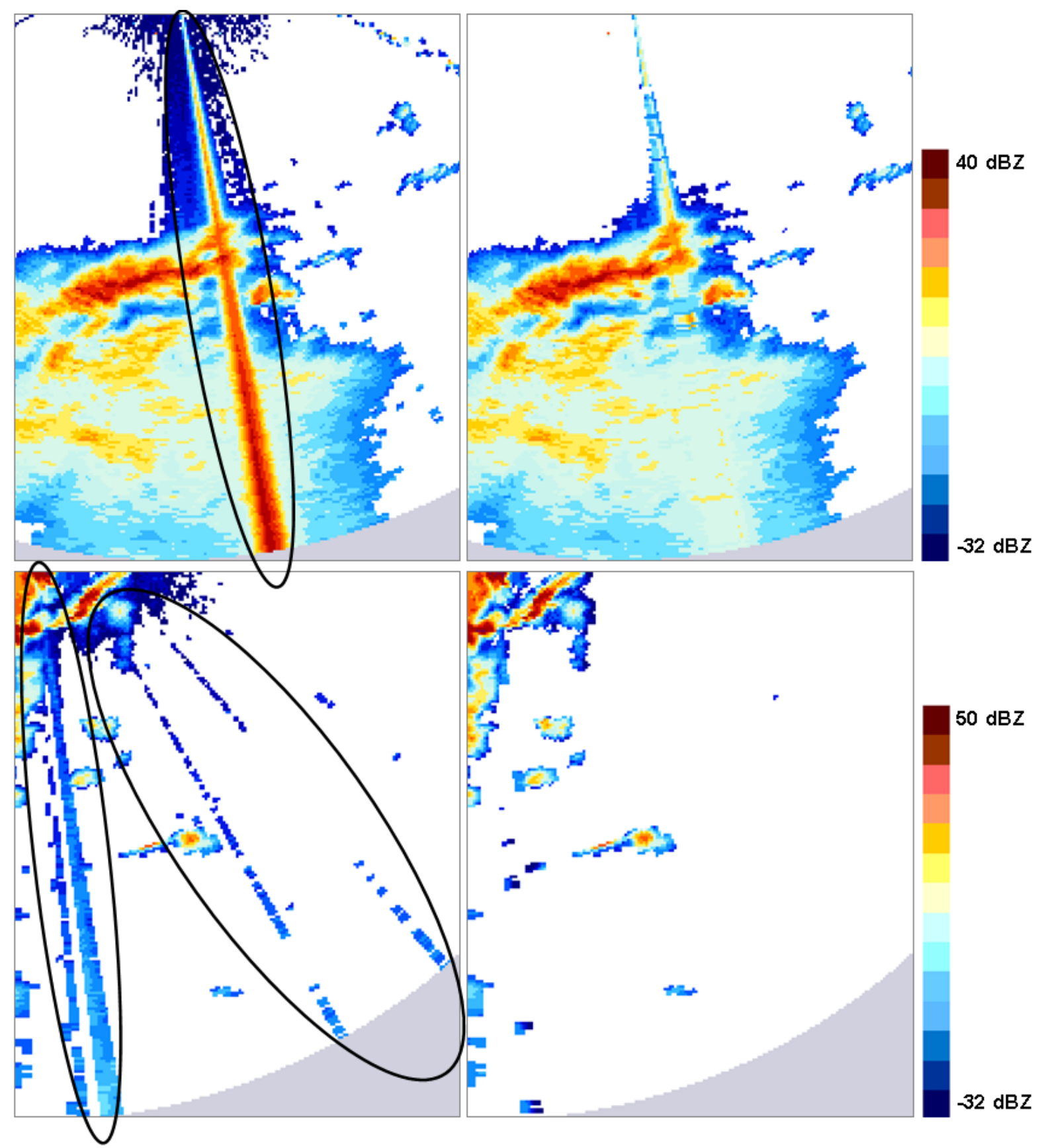

Figure 2. Examples of "wide" (upper row, Legionowo, 5 May 2010, 2200 UTC) and "narrow" (lower row, Legionowo radar, 10 May 2010 , 1530 UTC) spikes before and after corrections

$u_{7}(\alpha, l) \cdot u_{h}(\alpha, l)>0.2 \Rightarrow$

$\Rightarrow$ non - metorological echo in gate $(\alpha, l)$

"High" spurious echoes are all echoes detected at altitudes higher than $20 \mathrm{~km}$ where it is not possible for any meteorological echo to exist. Echoes from gates fulfilling this condition are removed.

All gates found affected by non-meteorological echoes are regarded as contaminated and their quality $Q I_{\text {NMET }}$ is lowered to 0.75 .

\section{Speckle removal}

Weather radar data is often affected by measurement noise which results in isolated radar gates with or without echo - socalled speckles (Fig. 4).

The first sub-algorithm is employed to detect reverse speckles, i.e. isolated gates without radar echo surrounded by rainy gates. A certain grid around a given non-echo gate is considered (e.g. \pm 1 azimuth and bin) and a number of rainy gates is determined. If this number does not exceed the preset threshold, the gate is regarded the reverse speckle and the average reflectivity of all rainy gates in the grid is assigned to it. 

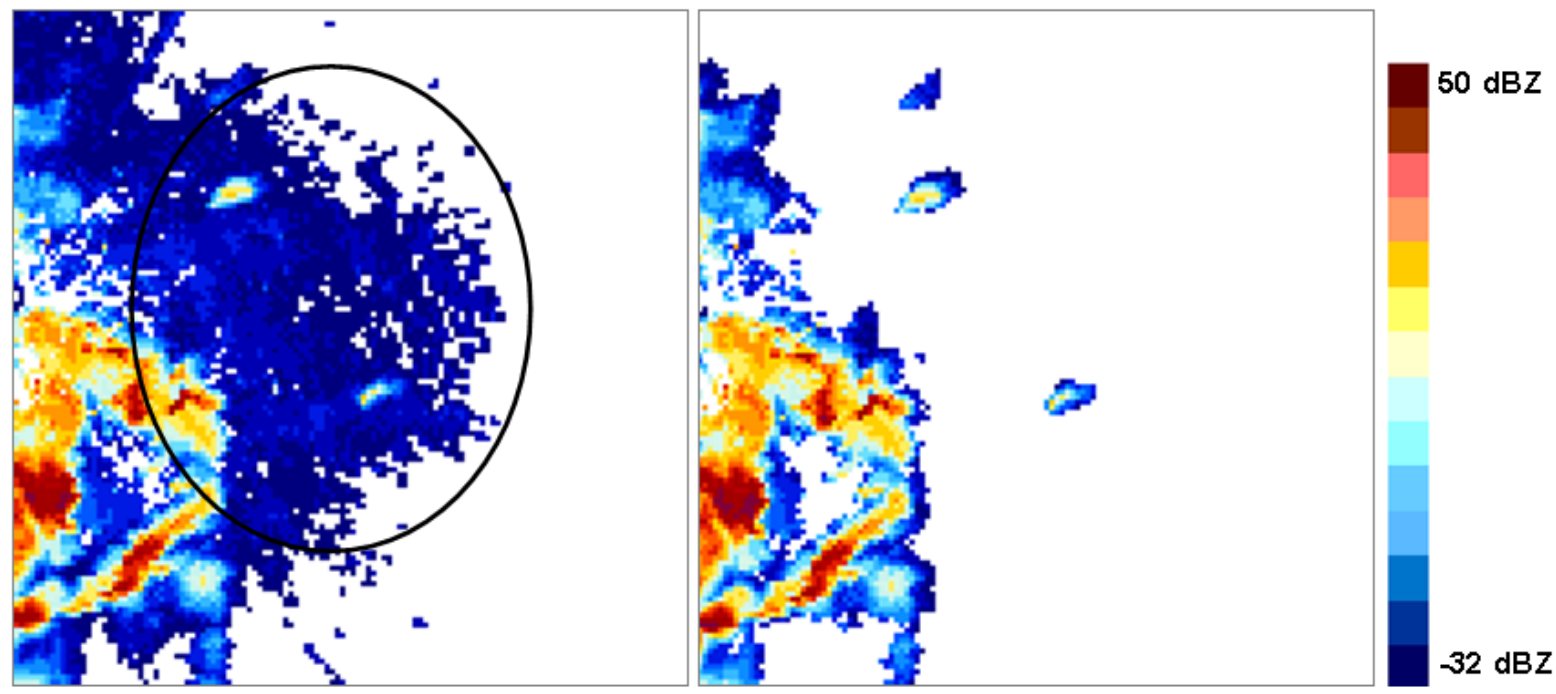

Figure 3. Example of non-meteorological echoes before and after correction (Legionowo, 10 May 2010, 1530 UTC)
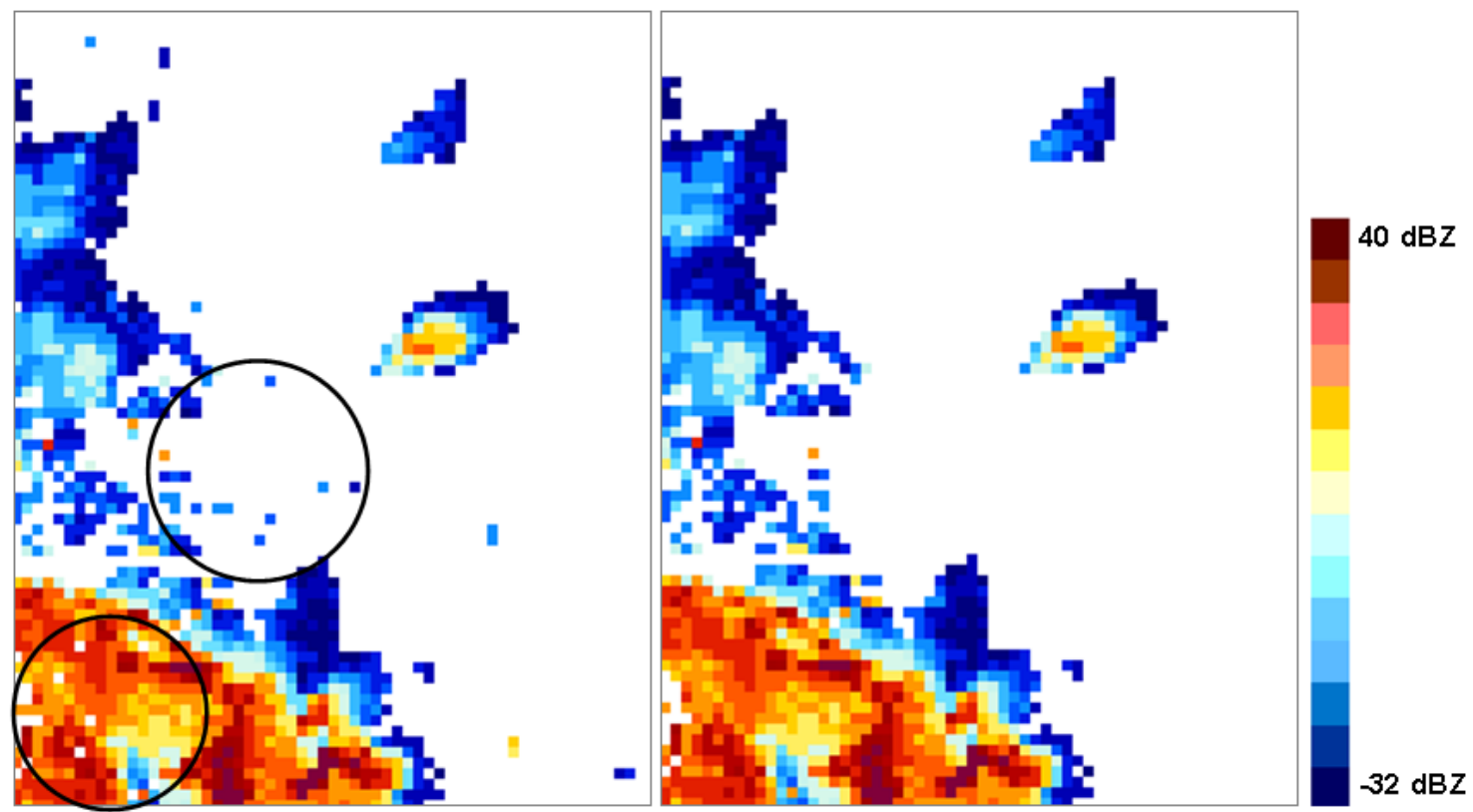

Figure 4. Example of speckle echoes before and after correction (Legionowo radar, 10 May 2010, 1530 UTC)

The next step is devoted to searching for ordinary speckles, i.e. isolated echoes on a non-echo background. The procedure is analogical - for a rainy gate the number of rainy gates within the surrounding grid is found and if the preset threshold is not achieved, the gate is considered a speckle and a non-echo value is assigned.

This algorithm is launched twice to clean the data more thoroughly. The quality $Q I_{S P E C K}$ in gates detected as speckles is reduced to 0.9 .

\section{Beam blockage and ground clutter}

If a radar beam hits any topographical object, it gets partially or completely blocked (Fig. 5). Assuming normal propagation conditions, the degree of partial beam blocking (PBB) can be computed from a digital terrain map taking account of the highest blocked point in the given beam cross-section:

$$
P B B=\frac{y \sqrt{r_{b}^{2}-y^{2}}+r_{b}^{2} \arcsin \frac{y}{r_{b}}+\frac{\pi r_{b}^{2}}{2}}{\pi r_{b}^{2}}
$$

where $r_{b}$ is the radius of the radar beam cross-section at the given distance from the radar site, and $y$ is the difference between the altitude of the terrain and the height of the radar beam centre (Bech et al. 2007).

Having estimated the $P B B$, radar reflectivity is corrected depending on the $P B B$ magnitude. If the $P B B$ exceeds the assumed threshold $a_{P B B}=0.7$ then reflectivity from a higher elevation is taken, but if such data is not available, the label "no data" is assigned (Eq. 7). If the PBB does not exceed the threshold, the correction is based on the $P B B$ : 

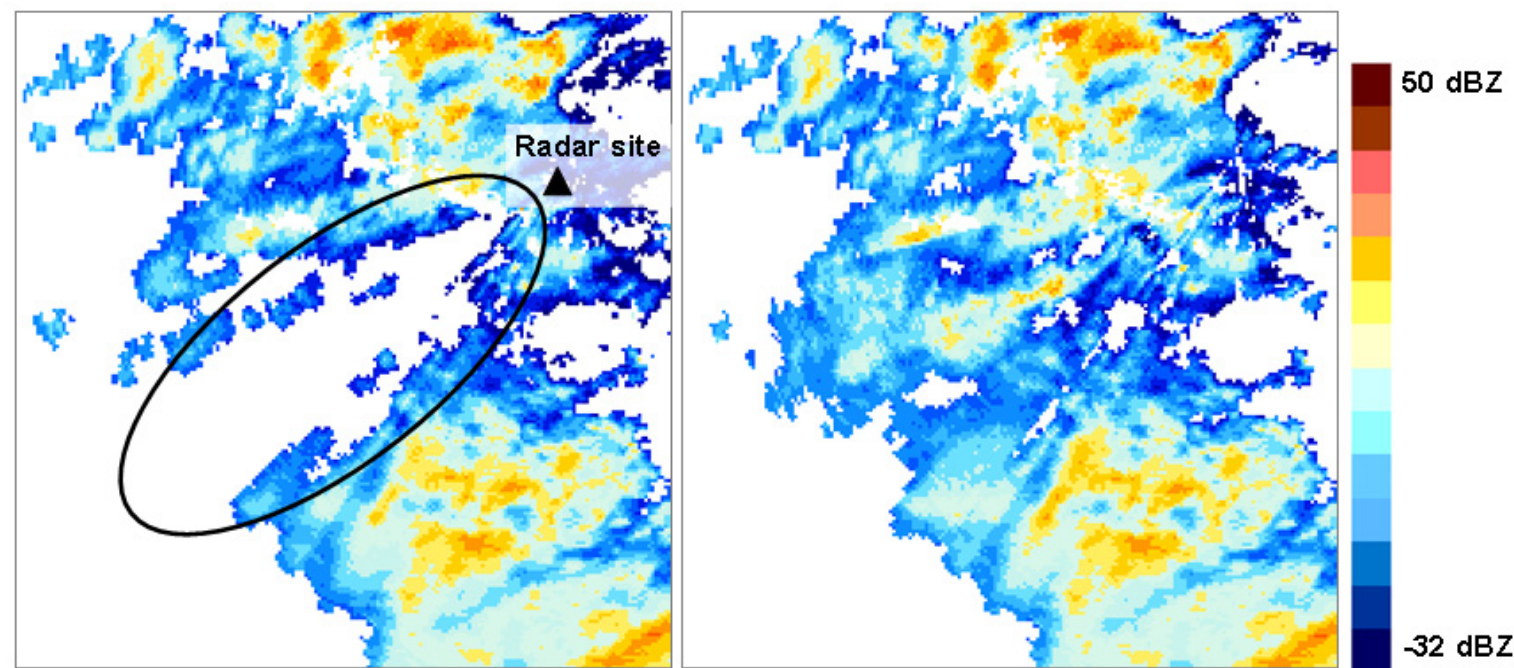

Figure 5. Example of radar beam blockage in the Sudety Mountains before and after correction (Pastewnik radar, 5 May 2010, 1800 UTC)
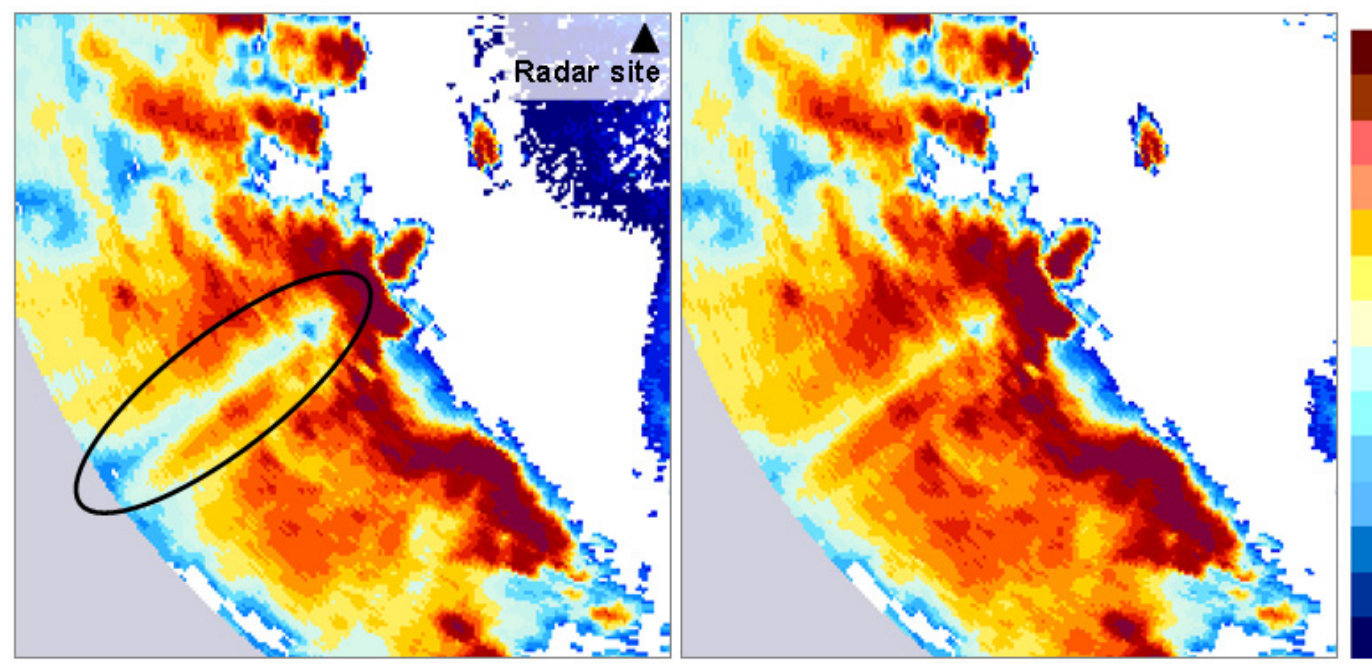

$50 \mathrm{dBZ}$

$32 \mathrm{dBZ}$

Figure 6. Example of radar beam attenuation in rain before and after correction (Legionowo radar, 16 August 2010, 1600 UTC)

$$
Z_{c o r}(e l)= \begin{cases}Z(e l)+10 \cdot \log _{10}(1-P B B)^{-1} & P B B \leq a_{P B B} \\ Z_{c o r}(e l+1) & P B B>a_{P B B} \text { and } e l \neq e l_{\text {max }} \\ \text { "no data" } & P B B>a_{P B B} \text { and } e l=e l_{\text {max }}\end{cases}
$$

where el means the current elevation; (el+1) means the neighbouring higher elevation; $\mathrm{e}_{\max }$ means the highest elevation.

The quality of measurement corrupted by beam blockage dramatically decreases. It was assumed that the decrease in quality is proportional to the $P B B$. If reflectivity in a specific gate is replaced by reflectivity from a higher elevation then $Q I_{P B B}$ is taken from the higher one, but multiplied by a factor of $\left(1-a_{P B B}\right)$.

The correction due to contamination by ground clutter is commonly carried out at the level of the radar system software by means of the statistical or Doppler filter. In order to assess the relevant decrease in quality, the lacking information is retrieved based on $P B B$. A given gate is considered a ground clutter if increase in $P B B$ along the radar beam exceeds the preset threshold. The gate where ground clutter is detected is characterized by the lowered quality index: the quality $Q I_{P B B}$ is divided by a factor of two.
Attenuation in rain

Attenuation occurs when the radar signal interacts with the meteorological object though which the signal propagates. It results in a decrease in the radar signal power and an underestimation of the measured reflectivity (Fig. 6):

$A=10 \cdot \log _{10} \frac{Z_{\text {cor }}}{Z}$

where $A$ is the attenuation (in $\mathrm{dB}$ ); $Z_{\text {cor }}$ is the non-attenuated reflectivity (in $\mathrm{mm}^{6} \mathrm{~m}^{-3}$ ) and $Z$ is the measured one.

The developed algorithm for the estimation of non-attenuated reflectivity is based on an empirical formula for the specific attenuation. Assuming the Marshall-Palmer Z-R (reflectivityrainfall) relationship, the two-way specific attenuation $A_{i}$ (in $\mathrm{dB}$ $\mathrm{km}^{-1}$ ) in precipitation between measurement gates $i-1$ and $i$ can be estimated from the formula (valid for C-Band radar):

$A_{i}=0.0044 \cdot\left(\frac{1}{200} Z_{i}\right)^{\frac{1.17}{1.6}}$

where $Z_{i}$ is the radar reflectivity in the gate $i$ (in $\mathrm{mm}^{6} \mathrm{~m}^{-3}$ ). The algorithm is applied iteratively ("gate by gate"), i.e. for gate $i$ 

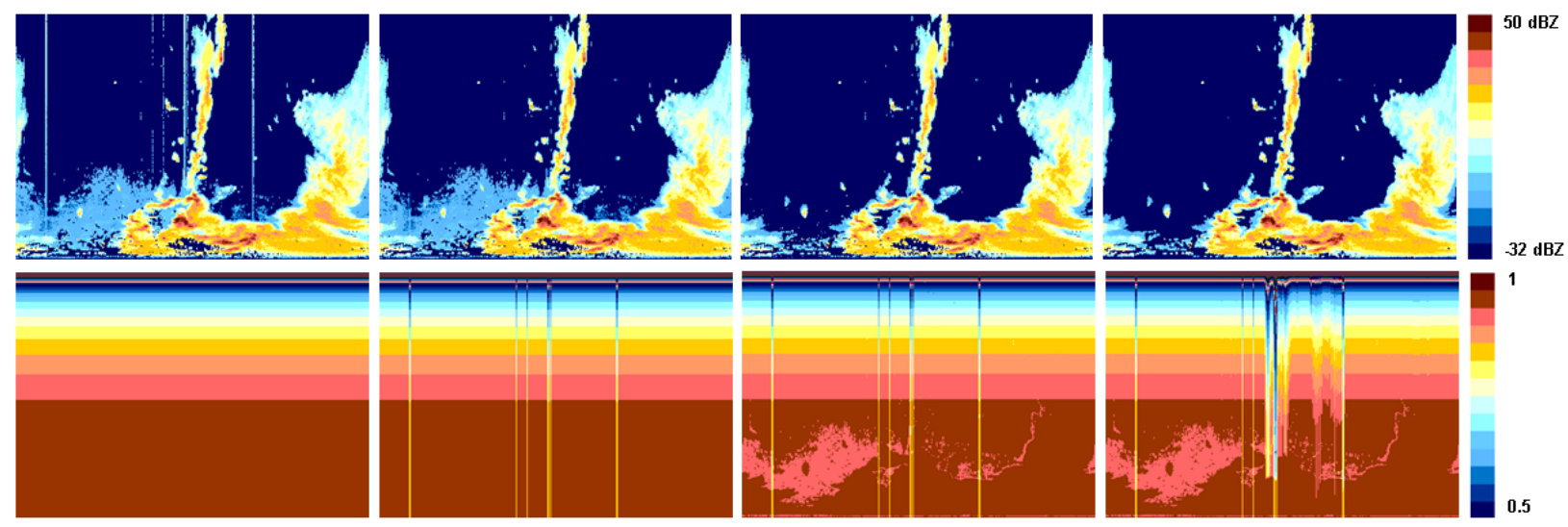

Figure 7. Example of quality control chain for the Legionowo radar (10 May 2010, 1530 UTC, elevation 0.5 , polar coordinates): reflectivity data (upper row) and QI fields (lower row), processed by the algorithms (from left to right): beam broadening; spike echo removal; non-meteorological echo and speckle removal; attenuation correction
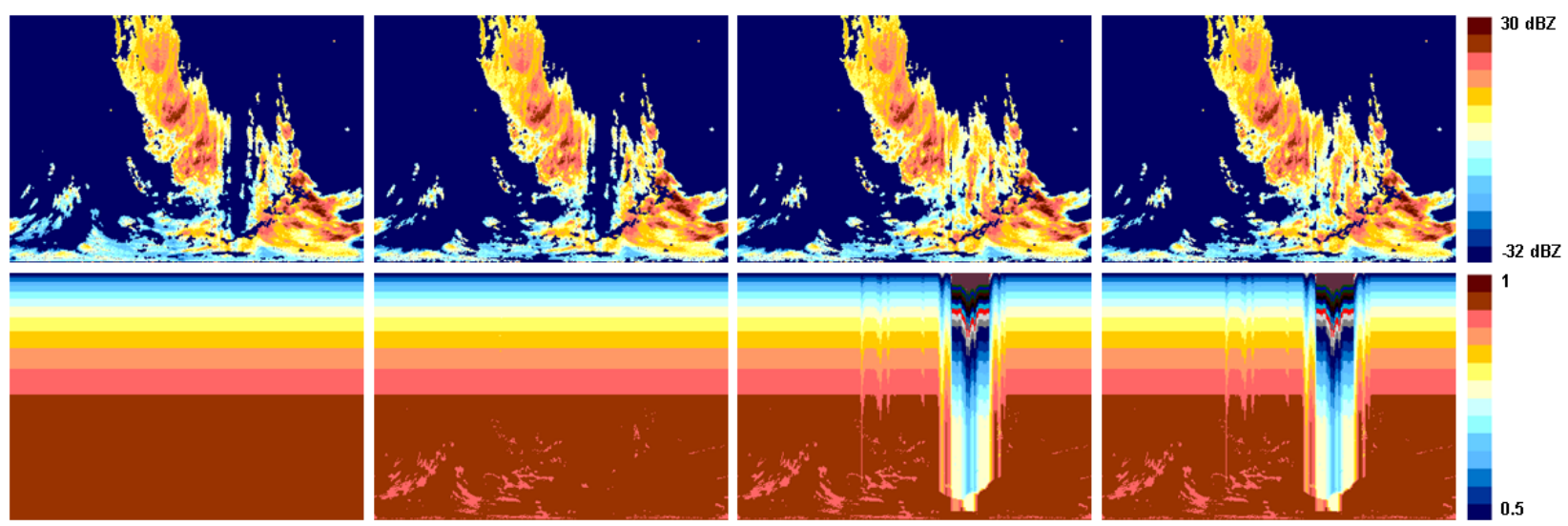

Figure 8. Example of quality control chain for the Pastewnik radar (5 May 2010, 1800 UTC, elevation 0.5 , polar coordinates): reflectivity data (upper row) and QI fields (lower row), processed by the algorithms (from left to right): beam broadening; non-meteorological echo and speckle removal; beam blockage; attenuation correction

along the radar beam the path-integrated attenuation $\left(P I A_{i}\right)$ is consecutively computed:

$$
P I A_{i}=\sum_{j=1}^{i} A_{j}
$$

and corrected reflectivity can be determined from:

$$
Z_{\text {icor }}=Z_{i} \cdot 10^{\frac{P I A_{i}}{10}}
$$

In order to avoid instability in the algorithm, the threshold values have to be set to limit the corrections for both specific attenuation $A_{i}$ and path-integrated attenuation $P I A_{i}$. The described algorithm is applied only for the gates with reflectivity higher than a preset threshold, otherwise the specific attenuation is assumed negligible.

The algorithm improves the data, but is not efficient enough in some cases. The more advanced algorithm designed for polarimetric radars seems more reliable; however, in the Polish radar network only two of eight radars employ such a technique. The relevant quality index $Q I_{A T T}$ for the considered gate $i$ is related to the magnitude of the path-integrated attenuation $P I A_{i}$ along the beam from the radar site to the gate. It is assumed that the quality index is perfect for the PIA, below the preset threshold, then decreases linearly to achieve zero for $P I A_{i}$ above the second threshold.
Total quality index

Computation of the total quality index $Q /$ for each gate of $3 D$ data volume is the final step in the estimation of 3D radar data quality. Having determined the individual quality indices characterizing data quality according to the particular quality factors described above, the total quality index $Q /$ is calculated by employing a multiplicative scheme:

$Q I=Q I_{B R O A D} \cdot Q I_{S P I K E} \cdot Q I_{N M E T} \cdot Q I_{S P E C K} \cdot Q I_{P B B} \cdot Q I_{A T T}$

The total $Q /$ field constitutes important information for the applications of weather radar data. Dealing with the uncertainty expressed by the index is a challenging issue. In the approach employed in this work a certain threshold for $Q I$ is preset: data of quality below the threshold is not accepted for application.

\section{Examples}

In Figs. 7 and 8 the results of the quality processing chain are presented as sequences of reflectivity fields along with the relevant $Q /$ fields (derived from the multiplicative scheme including all previous $Q$ I fields) for selected algorithms whose impacts are the most evident. Fields for the lowest elevation are depicted as they are usually burdened with errors most dramatically. In the first set for the Legionowo radar the strong contamination of spike echoes is observed, whereas ground clutter and related blockage is noticeable on data from the Pastewnik radar. 
Forecasts: $1200+0030$
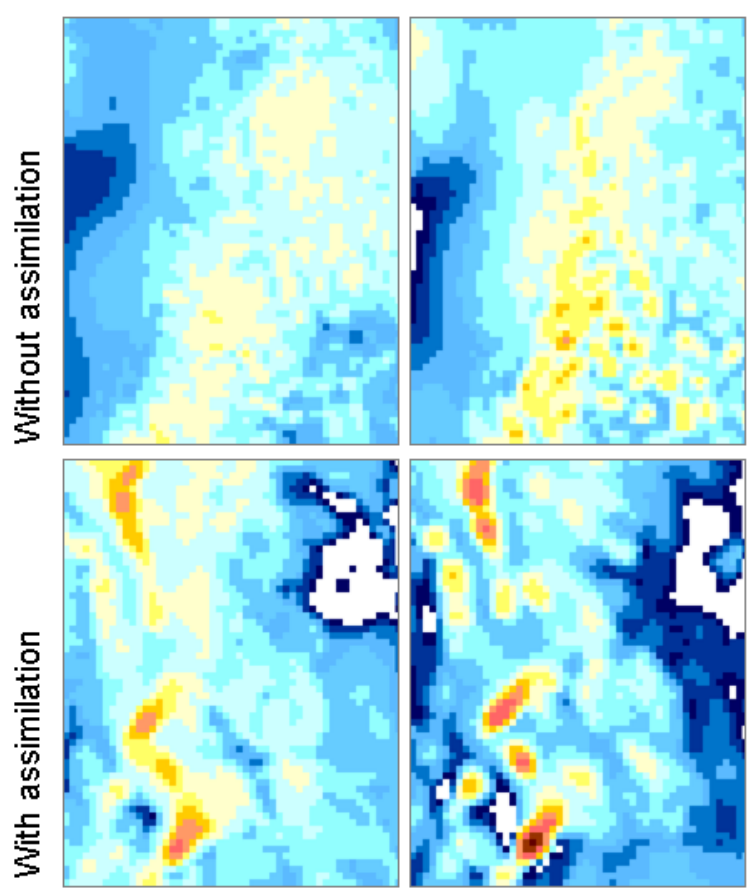

1300 UTC

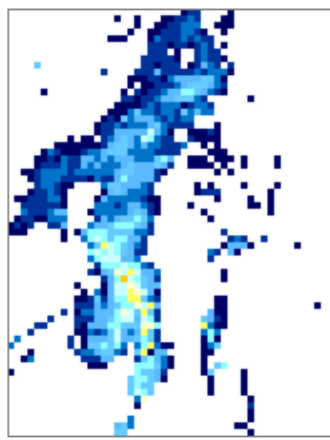

$1200+0130$
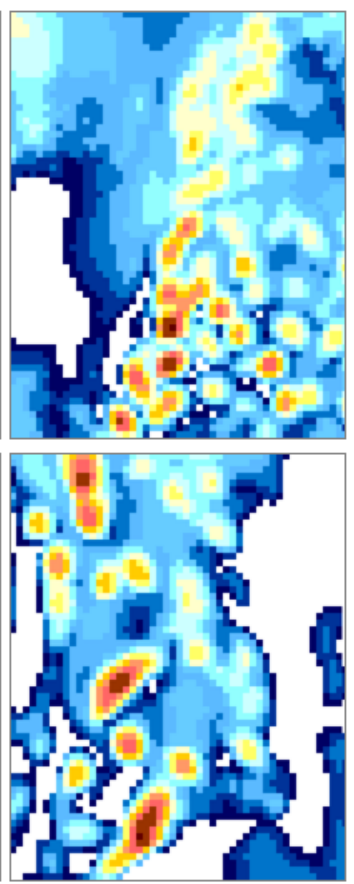

1330 UTC

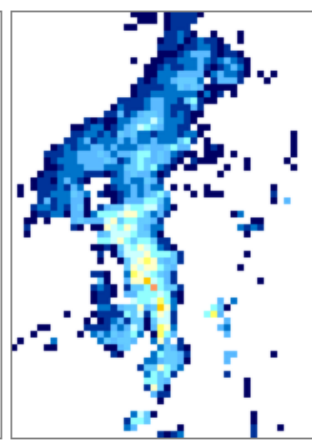

$1200+0200$ UTC

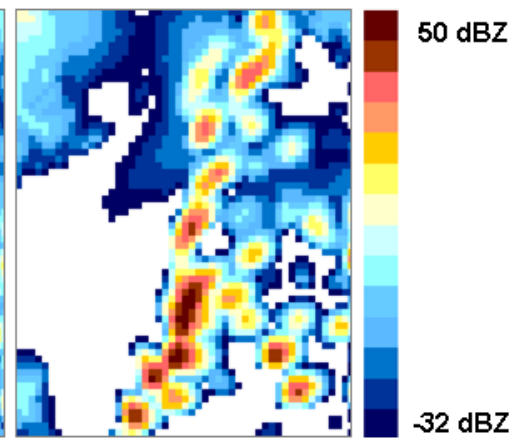

$50 \mathrm{dBZ}$

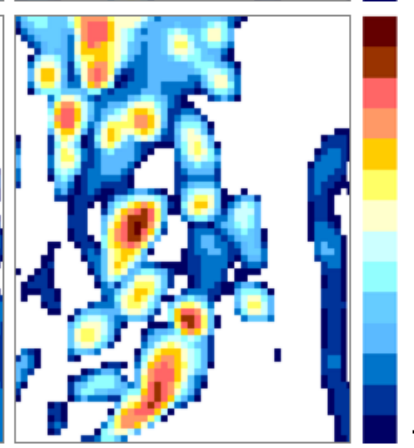

$32 \mathrm{dBZ}$

1400 UTC

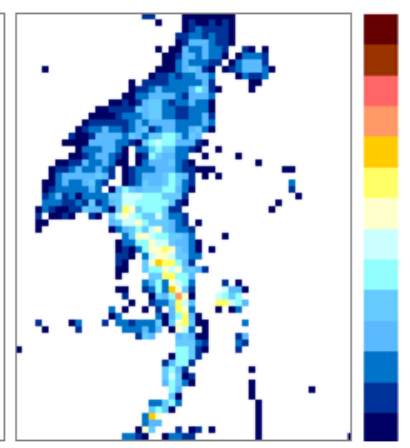

$32 \mathrm{dBZ}$

Figure 9. COAMPS model forecasts with and without radar data assimilation for lead times up to two hours, observed radar reflectivity (range of the Legionowo radar, 10 May 2010)

Radar reflectivity assimilation in the NWP model

The numerical weather prediction system chosen for this experiment is the NWP model COAMPS (Coupled Ocean/ Atmosphere Mesoscale Prediction System) developed in the Naval Research Laboratory. The atmosphere component of COAMPS is composed of the non-hydrostatic, fully compressible equations of motion. A full description of the model can be found in Chen et al. (2003). The model is used in the Interdisciplinary Centre for Mathematical and Computational Modelling at the University of Warsaw (ICM UW), both operationally and for research studies.

In the research the DART (Data Assimilation Research Testbed) system was employed to provide real-time, mesoscale analyses as the initial state of atmosphere for the COAMPSderived forecasts. The DART is an open source software program for ensemble data assimilation developed at the National Centre for Atmospheric Research.

A scheme of weather radar data assimilation using square root ensemble filters was investigated. The filter is formulated as a two-step procedure. The first step is a forecast of the model state to the next observation time. The ensemble members are evolved independently, according to the nonlinear weather prediction model. The second step is the analysis, i.e. adjustment of the entire ensemble to the information available in the new observations. Such an update reflects both the new state estimate and the uncertainty included in observations. The system of data assimilation transforms the ensemble of forecasts into an ensemble of analyses with statistics fitted to the observation data.

\section{Case study}

For the experiment, the case of heavy rainfall that occurred on 10 May 2010 in the range of the Legionowo radar was chosen. Almost all of Europe was under the influence of shallow low pressure areas, one with its centre over Scandinavia and the second moving from the Bay of Biscay towards France. The weather in Poland was mainly affected by a trough of low pressure between these two centres. During the day, convective precipitation organised in the narrow zone moved over Poland in a north-easterly direction (Fig. 9).

The model was run in the domain of 128 by 128 nodes with a grid resolution of $6 \mathrm{~km}$ in the horizontal axis and 40 vertical levels with variable resolution. The data was assimilated for two 

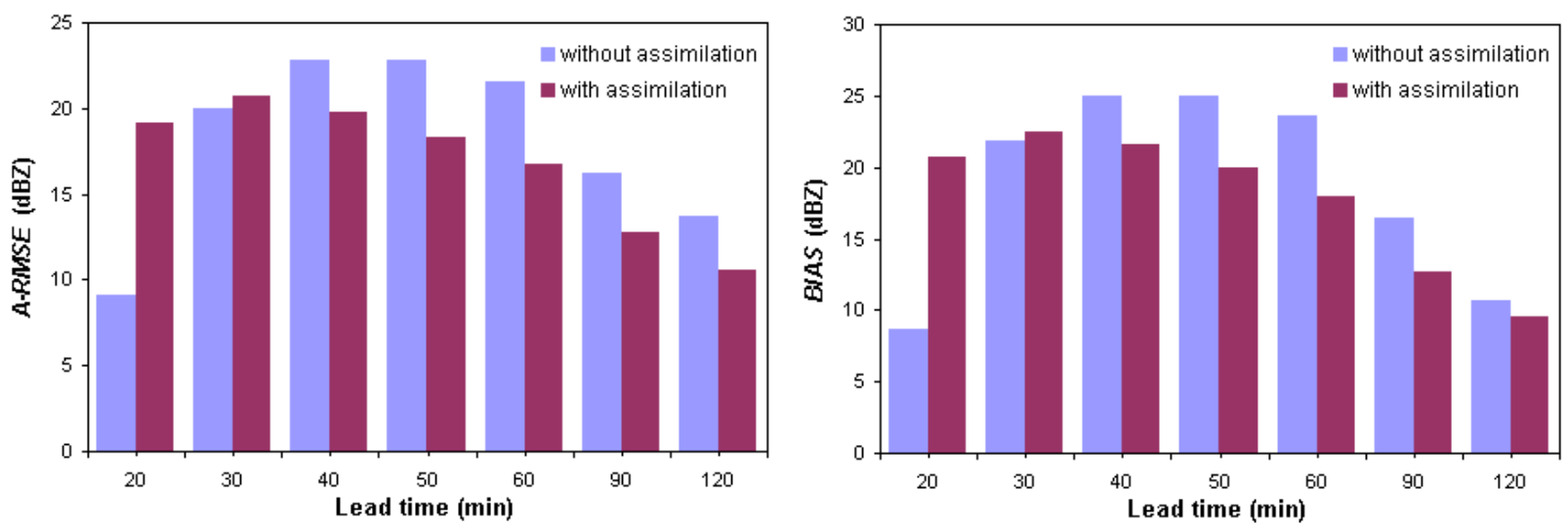

Figure 10. Diagrams of forecast quality criteria A-RMSE and BIAS, 10 May 2010

hours with a 10-minute time step, i.e. 13 assimilation cycles were performed.

Figure 9 shows examples of reflectivity data measured by the Legionowo radar on 10 May 2010, 1230-1400 UTC, and model forecasts with and without radar data assimilation generated at 1200 UTC with a lead time of up to 2 hours.

In order to evaluate the reliability of the assimilation algorithms, the observed radar reflectivity is considered a benchmark. The comparison between forecasts and observations is performed by employing different criteria, such as root mean square error, correlation coefficient, quality criteria obtained from the contingency table $(P O D$, probability of detection, and $F A R$, false alarm ratio, etc.), area-related A-RMSE (i.e. RMSE calculated for each pixel in the $9 \times 9 \mathrm{~km}$ grid around it, after sorting values in the grid in the descending order, then the mean for the whole image is calculated, Rezacova et al. 2007), and BIAS calculated from:

$B I A S=\frac{1}{N} \sum_{i=1}^{N}\left(F_{i}-O_{i}\right)$

where $F_{i}$ and $O_{i}$ are forecasted and observed reflectivity values (dBZ) respectively, for $i$-th pixel $(i=1, \ldots, N)$.

Figure 10 shows $A-R M S E$ and BIAS values for forecasts with and without radar data assimilation for a lead time of up to 2 hours.

Generally, A-RMSE and BIAS values indicate an improvement in forecast quality after radar data assimilation. A more detailed analysis shows that the model without assimilation overestimated the reflectivity field, while the assimilation slightly decreased this effect (smaller value of $B I A S$ ). High values of $P O D$ around 0.95 and $F A R$ around 0.75 indicate too large an area of the predicted reflectivity field.

The quality of forecasts measured by the correlation coefficient $R$ remains at a low level, although $R$ was almost doubled after taking account of assimilation. This shows large errors in predicting the location of precipitation, which is slightly improved after assimilation. The decrease in the A-RMSE error also suggests an improvement in the location of forecasted precipitation of high intensity.

The model COAMPS without assimilation had difficulty forecasting the precipitation zone correctly in space and time. The assimilation of radar data resulted in a slight improvement in the quality of the forecasts for lead times ranging from 0.5 to 2 hours, especially for precipitation location.

\section{Summary}

There is a potential for weather radar reflectivity as a source of information on the current atmospheric condition, which is very important for NWP models, especially in a convective scale. However, the radar observations should undergo a comprehensive data quality control prior to assimilation into the model. For this task the radar data processing chain was designed and developed to obtain as reliable radar observations as possible. The chain consists of the following correction algorithms: removal of non-meteorological echoes of different origin, such as signals from external antennas, side-lobe effects, biological objects, anomalous beam propagation, ground clutter, and additionally corrections due to beam blocking and shielding by terrain obstacles, attenuation in rain, etc. Another quality-related task is to characterize the quality of the data quantitatively, which is an important source of information for different applications.

The experiment with implementation of the radar reflectivity data after advanced corrections for assimilation in the COAMPS model was conducted on a heavy rainfall event as a case study. Ensemble Kalman filtering was employed as the technique for data assimilation. The COAMPS model without assimilation predicted convective precipitation in space and time rather poorly. After radar data assimilation, forecasts with a lead time above 30 minutes improved, especially for precipitation location. The results are very preliminary, but can be treated as promising; however, there is a need for further research to optimize the assimilation scheme for different conditions, especially to improve forecasting of convective phenomena.

\section{Acknowledgements}

The research described in the paper was performed in the framework of the project N N307 467738 granted by the National Science Centre (Poland). The algorithms for data correction and quality characterization were partially developed within the BALTRAD+ project in the framework of the Baltic Sea Region Programme. Weather radar data from the POLRAD network was provided by IMGW-PIB and the COAMPS model was made available by ICM UW. 
Bebbington, D, Rae, S, Bech, J, Codina, B \& Picanyol, M 2007, 'Modelling of weather radar echoes from anomalous propagation using a hybrid parabolic equation method and NWP model data', Natural Hazards and Earth System Sciences, vol. 7, pp. 391-398.

Bech, J, Gjertsen, U \& Haase, G 2007, 'Modelling weather radar beam propagation and topographical blockage at northern high latitudes', Q. J. R. Meteorol. Soc., vol. 133, pp. 11911204.

Chen, S, Cummings, J, Doyle, J, Hodur, RH, Holt, T, Liou, C, Liu, M, Mirin, A, Ridout, J, Schmidt, JM, Sugiyama, G \& Thompson, WT 2003, 'COAMPS 3.0 model description - General theory and equations', NRL Tech. Note NRL/ PUB/7500-0-3-448.

Einfalt, T, Szturc, J \& Ośródka, K 2010, 'The quality index for radar precipitation data: a tower of Babel?', Atmos. Sci. Let., vol. 11, pp. 139-144.

Gekat, F, Meischner, P, Friedrich, K, Hagen, M, Koistinen, J, Michelson, DB \& Huuskonen, A 2004, 'The state of weather radar operations, networks and products' in Weather Radar: Principles and Advanced Applications, ed P Meischner, Springer-Verlag, Berlin - Heidelberg, pp. 1-51.

Germann, U \& Joss, J 2004, 'Operational measurement of precipitation in mountainous terrain' in Weather Radar: Principles and Advanced Applications, ed $\mathrm{P}$ Meischner, Springer-Verlag, Berlin - Heidelberg, pp. 52-77.

Houtekamer, PL, Mitchell, HL, Pellerin, G, Buehner, M, Charron, M, Spacek, L \& Hansen, B 2005, 'Atmospheric data assimilation with an ensemble Kalman filter: results with real observations', Mon. Wea. Rev., vol. 133, pp. 604-620.

Meischner, P (ed.) 2004, Weather Radar: Principles and Advanced Applications. Springer-Verlag, Berlin - Heidelberg.
Michelson, D, Einfalt, T, Holleman, I, Gjertsen, U, Friedrich, K, Haase, G, Lindskog, M \& Jurczyk, A 2005, Weather radar data quality in Europe - quality control and characterization. COST Action 717, Working document, Luxembourg.

Ośródka, K, Szturc, J \& Jurczyk, A 2014, 'Chain of data quality algorithms for 3-D single-polarization radar reflectivity (RADVOL-QC system)', Meteorol. Appl. vol. 21, pp. 256-270.

Rezacova, D, Sokol, Z \& Pesice, P 2007, 'A radar-based verification of precipitation forecast for local convective storms', Atmospheric Research, vol. 83, pp. 211-224.

Šálek, M, Cheze, J-L, Handwerker, J, Delobbe, L \& Uijlenhoet, R 2004, Radar techniques for identifying precipitation type and estimating quantity of precipitation. COST Action 717, Working Group 1 - A review. Luxembourg,.

Sun, J 2005, 'Convective-scale assimilation of radar data: Progress and challenges', Q. J. R. Meteorol. Soc., vol. 131, pp. 3439-3463.

Villarini, G \& Krajewski, WF 2010, 'Review of the different sources of uncertainty in single polarization radar-based estimates of rainfall', Surveys in Geophysics, vol. 31, pp. 107-129.

Xue, M, Jung, Y \& Zhang, G 2010, 'State estimation of convective storms with a two-moment microphysics scheme and an ensemble Kalman filter: Experiments with simulated radar data', Q. J. R. Meteorol. Soc., vol. 136, pp. 685-700.

Zappa, M, Beven, KJ, Bruen, M, Cofiño, AS, Kok, K, Martin, E, Nurmi, P, Orfila, B, Roulin, E, Schröter, K, Seed, A. Szturc, J, Vehviläinen, B, Germann, U \& Rossa, A 2010, 'Propagation of uncertainty from observing systems and NWP into hydrological models: COST-731 Working Group 2', Atmos. Sci. Lett., vol. 11, pp. 83-91. 